\title{
RETRACTED ARTICLE: Downregulation of miR-133b/miR-503 acts as efficient prognostic and diagnostic factors in patients with osteosarcoma and these predictor biomarkers are correlated with overall survival
}

\author{
Seyad Alireza Bassampour ${ }^{1} \cdot$ Reza Abdi $^{2} \cdot$ Reza Bahador $^{2} \cdot$ Mohammadreza Shakeri $^{2}$. \\ Ali Torkaman ${ }^{3} \cdot$ Emad Yahaghi $^{4} \cdot$ Afshin Taheriazam $^{5}$
}

Published online: 16 August 2015

(C) International Society of Oncology and BioMarkers (ISOBM) 2016

\section{RETRACTED ARTICLE: Tumor Biol.}

DOI 10.1007/s13277-015-3918-9

This article has been retracted at the request of the Editor-in-Chief, the International Society of Oncology and BioMarkers (ISOBM) and the Publisher per the Committee on Publication Ethics guidelines. The article shows evidence of irregularities in authorship, there is strong reason to believe that the peer review process was compromised and the article is showing similarities with the following articles which were all submitted within a close timeframe:

Alireza Mirghasemi, Afshin Taheriazam, Seyyed Hasan Karbasy, Ali Torkaman, Mohammadreza Shakeri,

Electronic supplementary material The online version of this article (doi:10.1007/s13277-015-3918-9.) contains supplementary material, which is available to authorized users.

Afshin Taheriazam

afshintaheriazam@yahoo.com

1 Department of Orthopedics, AJA University of Medical Sciences, Tehran, Iran

2 Department of Orthopaedic and Trauma Surgery, Birjand University of Medical Sciences, Birjand, Iran

3 Department of Orthopedics, Firoozgar Hospital, Iran University of Medical Sciences, Tehran, Iran

4 Department of Molecular Biology, Baqiyatallah University of Medical Sciences, Tehran, Iran

5 Department of Orthopedics Surgery, Tehran Medical Branch, Islamic Azad University, Tehran, Iran
Emad Yahaghi and Aram Mokarizadeh, Down regulation of miR-133a and miR-539 are associated with unfavorable prognosis in patients suffering from osteosarcoma, Cancel Cell International (2015) DOI 10.1186/s12935015-0237-6

Date received: 23 July 2015

Seyyed Hasan Karbasy, Afshin Taheriazam, Alireza Mirghasemi, Farnoush Sedaghati, Mohammadreza Shakeri, Emad Yahaghi, Reza Bahador, Upregulation of miR-300 and downregulation of miR-125b act as potential predictor biomarkers in progression, metastasis, and poor prognosis of osteosarcoma. Tumor Biol. (2015) DOI:10. 1007/s13277-015-4000-3

Date received: 24 July 2015

The retracted paper was received 20 July 2015.

As such the validity of the content of this article cannot be verified.

The online version of this article contains the full text of the retracted article as electronic supplementary material. 